Z Gerontol Geriat 2017 · 50:273-274

DOI 10.1007/s00391-017-1240-4

Online publiziert: 11. Mai 2017

c) Springer Medizin Verlag GmbH 2017

CrossMark

\author{
Gerhard Igl $\left.\right|^{1,5} \cdot$ Thomas Klie $^{2,6} \cdot$ Frank Oswald $^{3} \cdot$ Gisela Zenz $^{4}$ \\ ${ }^{1}$ Institut für Sozialrecht und Gesundheitsrecht, Rechtswissenschaftliche Fakultät, Christian-Albrechts- \\ Universität zu Kiel, Kiel, Deutschland \\ ${ }^{2}$ Öffentliches Recht und Verwaltungswissenschaft, Evangelische Hochschule Freiburg, Freiburg, \\ Deutschland \\ ${ }^{3}$ Interdisziplinäre Alternswissenschaft (IAW), Fachbereich Erziehungswissenschaften, Frankfurter Forum \\ für interdisziplinäre Alternsforschung (FFIA), Goethe-Universität Frankfurt am Main, Frankfurt am Main, \\ Deutschland \\ ${ }^{4}$ Fachbereich Erziehungswissenschaften, Fachbereich Rechtswissenschaft, Frankfurter Forum für \\ interdisziplinäre Alternsforschung (FFIA), Goethe-Universität Frankfurt am Main, Frankfurt am Main, \\ Deutschland \\ ${ }^{5}$ Hamburg, Deutschland \\ ${ }^{6}$ Alpen Adria Universität Klagenfurt, IFF/Wien, Wien, Österreich
}

\title{
Rechtliche Aspekte des Alterns
}

Das Recht spielt für die Konstituierung der Lebensphase des höheren Alters als biografischer Lebensabschnitt und als soziale Zuschreibung - eine entscheidende Rolle. Beispielsweise gingen mit den im Laufe des 20. Jahrhunderts weitgehend verallgemeinerten Ruhestandsgrenzen auch Regelungsbedarfe für die Lebensphase Alter einher. In einem gewissen Gegensatz zur Bedeutung des Rechts für die gesellschaftliche Konstruktion von Alter steht die Befassung der Rechtswissenschaft mit dem Thema Recht. Für sie ist Alter ein junges Thema [2]. Seit Anfang der 1990er-Jahre rückt es mehr in den Blick der rechtswissenschaftlichen Aufmerksamkeit [4]. Mittlerweile hat sich die rechtswissenschaftliche Beschäftigung mit diesem Gebiet verbreitert und konsolidiert [1].

Rechtliche Belange älterer Menschen sind vielfältig und betreffen unterschiedliche, häufig alltägliche Lebensbereiche. Dazu gehören v.a. die Gebiete Alterssicherung, Teilhabesicherung und Altersdiskriminierung, Kranken- und Pflegeversicherung, Betreuungs- und Medizinrecht sowie das inzwischen zum Einrichtungs- und Dienstleistungsrecht weiterentwickelte Heimrecht. Aber auch verfassungs- und völkerrechtliche Fragen rücken mehr und mehr in den Fokus. Gleichwohl werden aus gerontologischer Sicht rechtliche Aspekte des Alterns teilweise nur unter sozialpolitischen Rahmenbedingungen erörtert (z. B. [5,
8]), was ihrer zentralen Bedeutung und Auswirkung auf den individuellen und gesellschaftlichen Alternsprozess nicht gerecht wird - man denke nur an die eingangs erwähnten Altersgrenzen [3, 6]. Denn, wenn es auch in Deutschland kein als solches kodifiziertes „Recht der älteren Menschen“ gibt, müssen neuere Entwicklungen ebenso wie die geltende Rechtspraxis oder offene Fragen und Rechtsschutzdefizite immer wieder neu herausgearbeitet und empirisch adressiert werden. So werden aktuell beispielsweise im Bereich des Rechtsschutzes älterer Menschen in der Pflege Vorschläge zum Schutz des Altenwohls erarbeitet (z. B. [8, 9]), die vor dem Hintergrund zukünftiger Herausforderungen der Versorgung in Deutschland ebenso wie im Lichte internationaler Forschung (z. B. [7]) in Wissenschaft und Praxis rezipiert und diskutiert werden sollten.

Daher werden in diesem Schwerpunkt einige ausgewählte rechtliche Aspekte des Alters und Alterns behandelt, ohne dabei einen Anspruch auf Vollständigkeit zu vertreten. Vielmehr adressieren die 4 ausgewählten Bereiche einen Querschnitt von thematischen „Dauerbrennern“ und aktuellen „hot topics" rechtswissenschaftlicher Alternsforschung.

Thomas Klie legt mit seinem Beitrag „Das Recht und die Lebensphase Alter“ zunächst den Grundstein für die Diskus- sion des Für und Wider eines „Altersrechts", indem er in die für das höhere Alter relevanten Rechtsgebiete und $\mathrm{Re}$ gelungen einführt und rechtliche Handlungsbedarfe beispielsweise für den besonderen Schutz vulnerabler Älterer diskutiert.

Claudia Mahler nimmt den internationalen Blick auf das Thema ein in ihrem Beitrag „Eine neue UN-Konvention zu den Rechten Älterer" und berichtet aus der UN-Arbeitsgruppe zum Schutz der Menschenrechte für ältere Personen. Themen sind die bessere Implementierung, die Schließung von Regelungslücken und eine Ausdifferenzierung der Rechte zur besseren staatlichen Umsetzung.

Gerhard Igl und Daniela Sulmann richten anlässlich des 10-jährigen Bestehens der Charta der Rechte hilfe- und pflegebedürftiger Menschen zunächst den Blick auf das rechtliche und sozialpolitische Umfeld des Entstehens der Charta („10 Jahre Pflege-Charta“). Sie gehen dann bilanzierend auf die Verbreitung und die Wirkungen der Charta ein und äußern sich zum Anpassungsund Veränderungsbedarf.

Anna Schwedler, Nadine Konopik und Kollegen/Kolleginnen gehen in der Folge auf eines der Problemfelder im Zusammenhang mit den Rechten hilfe- und pflegebedürftiger Menschen ein. Ihr Beitrag "Gewalt gegen alte Menschen in häuslicher Pflege" berichtet über Ziele und 
erste Befunde des Projekts „Menschenwürde und Persönlichkeitsrechte bei Versorgungsabhängigkeit in der häuslichen Pflege - Rechtsschutz für Pflegende und Pflegebedürftige“.

Julia Haberstroh und Tanja Müller wenden sich in ihrem Beitrag „Einwilligungsfähigkeit bei Demenz: interdisziplinäre Perspektiven“ der Handlungsund Entscheidungsunsicherheit in der rechtlichen sowie medizinischen Praxis aufgrund fehlender und interdisziplinär anerkannter Verfahren zur Beurteilung der Einwilligungsfähigkeit von Menschen mit Demenz zu und adressieren dieses Problem in einer empirischen Studie mit Experten aus den Feldern Recht, Medizin oder Medizinethik.

Dirk van den Heuvel und Carsten Klein schließlich berichten in ihrem Beitrag aus der aktuellen Rechtsprechung zu Fragestellungen aus Sicht des Bundesverbands Geriatrie. Damit ergänzen sie den Schwerpunkt um Einblicke in die für die Geriatrie relevante aktuelle Rechtsprechung der Landesschiedsstellen und des Bundessozialgerichts (und deren Folgen).

Insgesamt erlaubt die Auswahl dieser Beiträge einen Einblick in aktuelle und relevante Themen des vielfältigen Feldes „Rechtliche Aspekte des Alterns“, auch wenn es dessen Facettenreichtum nicht angemessen abbilden kann. Daher müssen wir uns dieser Thematik immer wieder aufs Neue zuwenden und ihre Verbindung $\mathrm{zu}$ anderen Bereichen der Gerontologie herausarbeiten.

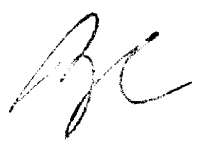

Prof. Dr. Gerhard Igl

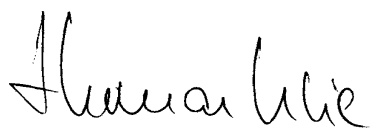

Prof. Dr. Thomas Klie

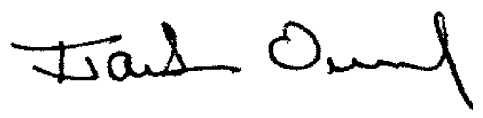

Prof. Dr. Frank Oswald

$$
\int .7 \text { enz }
$$

Prof. Dr. Dr. h.c. Gisela Zenz
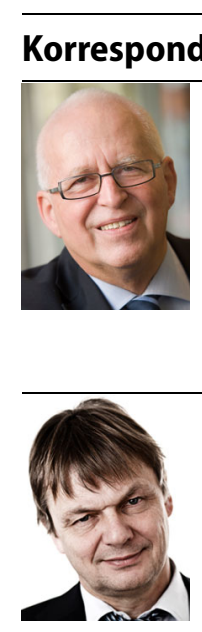

Prof. Dr. G. Igl

Institut für Sozialrecht und Gesundheitsrecht, Rechtswissenschaftliche Fakultät, Christian-AlbrechtsUniversität zu Kiel Kiel, Deutschland gerhard.igl@t-online.de

Prof. Dr. T. Klie

Öffentliches Recht und Verwaltungswissenschaft, Evangelische Hochschule Freiburg

Bugginger Str. 38, 79114 Freiburg, Deutschland klie@eh-freiburg.de

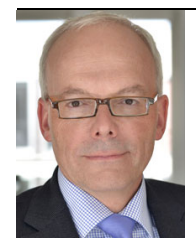

Prof. Dr. F. Oswald Interdisziplinäre Alternswissenschaft (IAW), Fachbereich Erziehungswissenschaften, Frankfurter Forum für interdisziplinäre Alternsforschung (FFIA), Goethe-Universität Frankfurt am Main Theodor-W.-Adorno-Platz 6, 60323 Frankfurt am Main, Deutschland oswald@em.uni-frankfurt.de

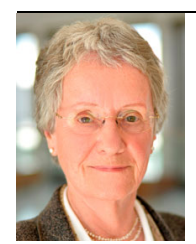

Prof. Dr. Dr. h.c. G. Zenz Fachbereich Erziehungswissenschaften, Fachbereich Rechtswissenschaft, Frankfurter Forum für interdisziplinäre Alternsforschung (FFIA), Goethe-Universität Frankfurt am Main Theodor-W.-Adorno-Platz 6, 60323 Frankfurt am Main, Deutschland gzenzffm@aol.com

Interessenkonflikt. G. Igl, T. Klie, F. Oswald und G. Zenz geben an, dass kein Interessenkonflikt besteht.

\section{Literatur}

1. Becker U, Roth M (Hrsg) (2013) Recht der Älteren. De Gruyter, Berlin Boston

2. Igl G (1993) Recht und Alter - ein diffuses Verhältnis. In: Becker B, Bull HP, Seewald O (Hrsg) Festschrift für Werner Thieme zum 70. Geburtstag. Heymanns, Köln Berlin München, S747-767

3. Igl G (2012) Höchstaltersgrenzen im Recht. In: Wahl H-W, Tesch-Römer C, Ziegelmann J
(Hrsg) Angewandte Gerontologie: Interventionen für ein gutes Altern in 100 Schlüsselbegriffen. Kohlhammer, Stuttgart, S154-159

4. Igl G, Klie T (Hrsg) (2007) Das Recht der älteren Menschen. Nomos, Baden-Baden

5. Klie T (2012) Pflegeversicherung. In: Wahl H-W, Tesch-Römer C, Ziegelmann J (Hrsg) Angewandte Gerontologie: Interventionen für ein gutes Altern in 100 Schlüsselbegriffen. Kohlhammer, Stuttgart, S148-153

6. Klie T (2013) Altersdiskriminierung und Altersgrenzen im Recht. In: Jähnichen $T$, Meireis $T$, Rehm J, Reuter H-R, Reihs S, Wegner G (Hrsg) Alternde Gesellschaft. Soziale Herausforderungen des längeren Lebens. Jahrbuch Sozialer Protestantismus, Bd. 6. Gütersloher Verlagshaus, Gütersloh, S207-245

7. Teresi J (2016) State of the science on prevention of elder abuse and lessons learned from child abuse and domestic violence prevention: toward a conceptual framework for research.J Jlder Abuse Negl 28(4-5):263-300

8. Zenz G (2014) Gewaltschutz im Alter - Ethik und Recht vor neuen Herausforderungen. In: Götz I, Schwenzer I, Seelmann K, Taupitz J (Hrsg) Familie - Recht - Ethik. Festschrift für Gerd Brudermüller zum 65. Geburtstag. Beck, München, S 953-962

9. Zenz G (2017) Gewalt in der Pflege alter Menschen - Rechtsschutz für Pflegende und Gepflegte. In: Brisch KH (Hrsg) Bindungstraumatisierungen. Wenn Bindungspersonen zu Tätern werden. KlettCotta, Stuttgart, S 140-153 